\title{
Identification of Suitable Reference Genes for qPCR Analysis of 4T1 Mouse Mammary Tumor Cell Line
}

Richa Arora', Waseem Akram Malla ${ }^{1}$, Arpit Tyagi², Shikha Saxena ${ }^{3}$, Sonalika Mahajan ${ }^{1}$, Basavaraj Sajjanar ${ }^{1}$, Ashok Kumar Tiwari ${ }^{4}$

10.18805/IJAR.B-4678

\begin{abstract}
Background: Identification of candidate reference genes for real time PCR study is a preliminary requirement to normalize experimental data and thus, deduce a reliable conclusion. Complex tissues like mouse mammary gland constitutes various cell types which makes it difficult to identify reference gene constantly expressing under different experimental conditions.

Methods: In this study we have identified suitable reference genes for 4T1 tumor cell line derived from mouse mammary tumor cells. We have studied four genes namely Gapdh, Actb, Prdx1 and Ctbp 1 for their expression stability in CPV2.NS1 post transfected 4T1 cells by Best Keeper.

Result: By our study, three reference genes i.e. Prdx1, Gapdh and Ctbp1 were found to be quite correlated with the BestKeeper index, but by considering all three criteria of selection by BestKeeper algorithm, Prdx 1 showed minimum standard deviation and coefficient of variation and was found to be ranked at first position by BestKeeper which suggests $\operatorname{Prdx} 1$ to be considered as better internal control gene among all other reference genes taken in our study for qPCR based experiments in 4T1 mouse mammary tumor cell line transfected with CPV2.NS1.

Key words: Canine parvovirus, Mammary gland tumor, Non-structural gene 1, Oncolytic viral genes, Pearson correlation coefficient, Standard deviation.
\end{abstract}

\section{INTRODUCTION}

Gene expression studies in complex tissues are challenging due to the diversity of the cell types that constitute such tissues, each type with a different gene expression profile. The mouse mammary gland is one of such complex tissue that contains various cell types depicting varying gene expression under different conditions Han et al. (2010). Mammary gland tumor is also an unusual physiological condition of the mammary gland in which there is an abnormal growth of cells and is quite possibly the most widely recognized oncologic disease in the world Sleeckx et al. (2011).

In both humans and animals, cancer incidences are increasing day by day. After skin cancer, breast cancer is frequently reported cancer in women Zaimy et al. (2017). Oncolytic viral gene therapy has recently gained significance for cancer treatment as all other traditional treatment modalities kill normal cells along with neoplastic cells. Therapeutic properties of various oncolytic genes (and their protein products) have been reported, which include VP3 (apoptin) of Chicken Infectious Anemia Virus (CIAV), E2 and E7 of Papillomaviruses; E1A 12S and 13S proteins, E3 and E4 of Adenovirus, tat of Human Immunodeficiency virus (HIV-1), Parvovirus NS1, Simian virus (SV40) large T antigen and Hemagglutinin-Neuraminidase (HN) protein of Newcastle Disease Virus (NDV). These genes, on ectopic expression, mediate targeted killing of tumor cells without causing harm to healthy cells Gupta et al. (2015). Non structural gene 1 of Canine Parvovirus 2 (CPV2.NS1) is one such gene reported to have oncolytic effect and causes apoptosis through
'Division of Veterinary Biotechnology, ICAR-Indian Veterinary Research Institute, Izatnagar-243 122, Uttar Pradesh, India.

${ }^{2}$ GB Pant University of Agriculture and Technology, Pantnagar-263 145, Uttarakhand, India

${ }^{3}$ Division of Animal Genetics, ICAR-Indian Veterinary Research Institute, Izatnagar-243 122, Uttar Pradesh, India.

${ }^{4}$ Central Avian Research Institute, ICAR-Indian Veterinary Research Institute, Izatnagar-243 122, Uttar Pradesh, India.

Corresponding Author: Richa Arora, Division of Veterinary Biotechnology, ICAR-Indian Veterinary Research Institute, Izatnagar-243 122, Uttar Pradesh, India.

Email: richaarora5521@gmail.com

How to cite this article: Arora, R., Malla, W.A., Tyagi, A., Saxena, S., Mahajan, S., Sajjanar, B. and Tiwari, A.K. (2021). Identification of Suitable Reference Genes for qPCR Analysis of 4T1 Mouse Mammary Tumor Cell Line. Indian Journal of Animal Research. DOI: $10.18805 /$ IJAR.B-4678.

Submitted: 15-06-2021 Accepted: 06-10-2021 Online: 03-11-2021

intrinsic/ mitochondrial pathway in cancer cell line and also induce anti-tumor immune response in various tumor models (Saxena et al., 2013; Santra et al., 2014; Gupta et al., 2016; Bhat et al., 2017). Quantitative real-time polymerase chain reaction ( $q R T-P C R$ ) has recently emerged as a powerful tool to study gene expression in bodily tissues including the mammary glands Han et al. (2010).

Despite being versatile and flexible, qRT-PCR suffers some limitations in the number of genes that can be studied together. High throughput sequencing has however come a 
long way to overcome the problems by identifying gene expression changes on a large scale and by generating reproducible results. RNA sequencing is one such clear cut measure to get accurate expression data with minimum variation but it requires validation by other methods. Given its high sensitivity, qRT-PCR is most commonly used for such validation purposes but an indispensable requirement with qPCR is the selection of suitable endogenous/reference genes to minimize variation Sahu et al. (2018). Reference/ Endogenous genes are those genes that are expressed uniformly in samples with different experimental conditions or treatments. Housekeeping genes are used mostly as internal control genes which normalize the variations in the qRT-PCR experiment (Tong et al., 2009; Köhsler et al., 2020). In the present study, the expression stability of four housekeeping genes namely Prdx1, Ctbp 1, Gapdh and Actb was compared in the 4T1 cell line (Mice mammary tumor cell line) transfected with oncolytic Non-structural gene 1 of Canine Parvovirus 2 (CPV2.NS1).

\section{MATERIALS AND METHODS}

This study was conducted in the month of January-February, 2021 in Indian Veterinary Research Institute (IVRI), Izatnagar, Bareilly, Uttar Pradesh.

\section{Cell culture and transfection}

4T1 cells were acquired from ATCC (American Type Culture Collection; Rockville, MD) and grown in RPMI 1640 media (supplemented with $5 \%$ FBS, penicillin $100 \mathrm{U} / \mathrm{ml}$, streptomycin $100 \mathrm{~g} / \mathrm{ml}$ and L-Glutamine) (DuchefaBiocheme, Netherlands) at $37^{\circ} \mathrm{C}$ under $5 \%$ CO2. The CPV2.NS1 gene was available as pVIVO1.NS1 in our laboratory Gupta et al. (2016). Transfection of 4T1 cells with pVIVO1.NS1 and pVIVO1 was carried out using Lipofectamine $₫ 3000$ as per the manufacturer's instructions. The empty vector pVIVO1transfected $4 \mathrm{~T} 1$ cells were used as control samples. Cell pellet for RNA isolation and GPCR were collected from both empty vector and pVIVO1.NS1 transfected $4 \mathrm{~T} 1$ cells at 24 $\mathrm{hr}$ and $48 \mathrm{hr}$ post-transfection.

\section{RNA extraction and cDNA synthesis}

Total RNA was extracted from cell pellets collected in RNAlater (QIAGEN, Germany) using TRIzol reagent (Thermo Scientific, USA) as per manufacturer's guidelines. The RNA concentration and integrity was examined by
Nanodrop spectrophotometer. The purity of all RNA samples was assessed by the absorbance ratios of OD260/280 and OD260/230. RevertAid TM First Strand cDNA Synthesis Kit (Thermo Fisher Scientific Inc, USA) was used for preparing cDNA from one microgram of RNA of each sample following the protocol provided in the product manual. The cDNA thus prepared were stored at $-20^{\circ} \mathrm{C}$ till further use. The cDNA was diluted 10-fold for subsequent qRT-PCR analysis.

\section{Primer designing and sequence of reference genes}

Sequences for designing primers for all four reference genes were obtained from $\mathrm{NCBI}$ and primers were prepared by using software Primer Quest (https://www.idtdna.com). Primers for quality parameters were verified in Oligo Analyzer and NCBI Primer-BLAST. The sequences of all four primers are given in (Table 1).

\section{Quantitative Real-time PCR (qRT-PCR)}

Real time PCR was performed with Maxima SYBR Green qPCRMasterMix (Thermo Fisher Scientific Inc.,USA) using an Agilent AriaMx Real-Time PCR System. The thermal profile of the reaction started initially with $50^{\circ} \mathrm{C}$ for $2 \mathrm{~min}$, $95^{\circ} \mathrm{C}$ for $10 \mathrm{~min}$, followed by 40 cycles at $95^{\circ} \mathrm{C}$ for $15 \mathrm{~s}, 56^{\circ} \mathrm{C}$ for $30 \mathrm{~s}$ and $72^{\circ} \mathrm{C}$ for $30 \mathrm{~s}$. Each reaction included $5 \mu$ of $2 \mathrm{X}$ SYBR Green Master mix, $0.1 \mu \mathrm{l}(10 \mathrm{pmol})$ of endogenous control forward and reverse primer, $10 \mathrm{ng}$ of CDNA and NFW to make up the volume to $10 \mu \mathrm{l}$. Finally, a melting curve was generated by increasing temperature from $95^{\circ} \mathrm{C}$ to $65^{\circ} \mathrm{C}$ for $30 \mathrm{sec}$ each, followed by the final step of $95^{\circ} \mathrm{C}$ for $30 \mathrm{sec}$.

\section{Gene expression stability analysis}

Gene expression stability analysis of reference genes was conducted using the BestKeeper algorithm available at RefFinder (https:www.heartcure.com.au/reffinder/). Direct Cycle threshold (ct) values of samples were used as an input for the web-based tool RefFinder Xie et al. (2012).

\section{RESULTS AND DISCUSSION}

Real-time PCR is a highly sensitive method for absolute and relative quantification of gene expression. The reliability of QPCR data would further be greatly increased by including endogenous genes whose transcription level would be unwavering in the diverse experimental situations. Several approaches have been used to discover steadiness in gene expression and choose the best endogenous genes in the

Table 1: Primer Sequences of four reference gene used in validation study.

\begin{tabular}{|c|c|c|c|}
\hline Primer & Gene symbol & Primer sequence & Size of product \\
\hline \multirow[t]{2}{*}{ Glyceraldehyde 3-phosphate dehydrogenase } & Gapdh & F.P - GTATGACTCCACTCACG & $150 \mathrm{bp}$ \\
\hline & & R.P -GACTCCACGACATACTC & \\
\hline \multirow[t]{2}{*}{ Peroxiredoxin 1} & $\operatorname{Prd} x 1$ & F.P -ACCTGGCAGTGATACCATCAAG & $113 \mathrm{bp}$ \\
\hline & & R.P-TTCTTCTGGCTGCTCAATGC & \\
\hline \multirow[t]{2}{*}{ Beta-actin } & $A c t b$ & F.P -GAATGGGTCAGAAGGAC & $122 \mathrm{bp}$ \\
\hline & & R.P - CCAGATCTTCTCCATGTC & \\
\hline \multirow[t]{2}{*}{ C-terminal binding protein } & Ctbp 1 & F.P -TGCCACATCCTGAACCTGTAC & $147 \mathrm{bp}$ \\
\hline & & R.P - TAGTCCAATGATGCCCAAGGTC & \\
\hline
\end{tabular}


context of the relevant experimental conditions, but to date, there is no consensus on which technique we should rely on to study internal control gene expression stability. Among all four reference genes taken in our study, Actb (cytoskeletal structural protein- Beta actin) and Gapdh (glycolytic enzymeGlyceraldehyde 3- phosphate dehydrogenase) are traditional reference genes that are usually used for qPCR experiments whereas $\operatorname{Prd} x 1$ (peroxiredoxins) and Ctbp 1 (Cterminal binding protein 1 ) are recently reported internal control genes for conducting qPCR based mouse mammary gland experimental studies (van de Moosdijk and van Amerongen, 2016).

For our qPCR data normalization in CPV2.NS1 transfected 4T1 mice mammary gland tumor cell line, we compared four housekeeping genes i.e. Gapdh, Prdx1, Ctbp1 and Actb by the BestKeeper algorithm. BestKeeper calculates variation in gene expression for each reference gene based on Crossing points (CP) which is the number of cycles required to cross selected threshold fluorescence Mehta et al. (2010). On basis of these CP values, BestKeeper calculates Standard deviation (SD) and Coefficient of variation (CV) for all housekeeping genes. By performing various pairwise correlation analyses, this expression analysis tool estimates relationships among all possible pairs of reference genes by using raw Ct values of each gene Pfaffl et al. (2004). Standard deviation (SD) of the endogenous genes is a key aspect to identify genes with maximum stability by BestKeeper software; the higher the Coefficient of variation (CV) and Standard Deviation (SD) values, the more unstable the reference gene Bao et al. (2019). BestKeeper algorithm also assesses the correlation coefficient $(r)$ of each reference gene under study to the BestKeeper index, as calculated from the geometric mean of the remaining reference genes. Generally, reference gene with SD values $>1$ are considered unstable and should be

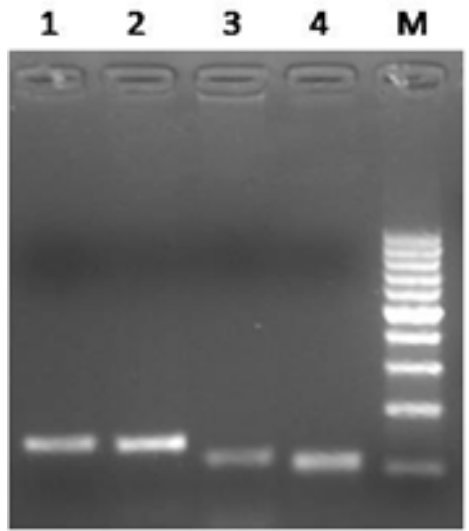

Fig 1: Amplication of four reference genes depicted in agarose gel. 1. Amplification of Gapdh

2. Amplification of Ctbp1

3. Amplification of Actb

4. Amplification of $\operatorname{Prdx} 1$

M. 100 bp ladder sorted out. So, overall three indicators are provided by BestKeeper to identify expression stability of a housekeeping gene which are CV, SD and correlation coefficient (r).

Initially, four endogenous genes were amplified in the samples examined and single-band products of appropriate size were obtained by agarose gel electrophoresis (Fig 1). All genes in qRT-PCR assays produced one single peak in the melt curve analysis (Fig 2). After generating melt curve, average $\mathrm{Ct}$ values of triplicates of each reference gene were used for visualizing the reference gene showing stable expression and Prdx1 Ct values were found to be varying less among both 24 and $48 \mathrm{hr}$ post transfection in both control and treatment groups as compared to all other three reference gene in our study (Fig 3 ).

BestKeeper uses raw $\mathrm{Ct}$ values of all genes as input. Analysis of similar data with BestKeeper begins with the estimation of variations i.e., SD and CV for all the four candidate endogenous genes in the samples depicted in (Tables 2 and 3 ). In the case of 4 T1 cells with $24 \mathrm{hr}$ posttransfection, not a single housekeeping genes under study showed an SD>1 indicating that all of the genes under study were appropriate enough to be considered for selecting them as endogenous genes. But in $48 \mathrm{hr}$ post-transfection, Actb had SD more than 1 which eliminates this reference gene from our consideration.

Table 2: Crossing Points (CP) data of housekeeping genes by Best Keeper for $24 \mathrm{mhr}$ post. transfection.

\begin{tabular}{lcccc}
\hline & Ctbp1 & Gapdh & Prdx1 & Actb \\
\cline { 2 - 5 } $\mathrm{N}$ & 6 & 6 & 6 & 6 \\
\hline geo mean [CP] & 21.86 & 17.14 & 21.56 & 16.47 \\
AR mean [CP] & 21.87 & 17.16 & 21.57 & 16.50 \\
min [CP] & 21.15 & 16.53 & 20.80 & 15.46 \\
max [CP] & 22.61 & 17.87 & 22.24 & 17.49 \\
std dev [+/- CP] & 0.70 & 0.59 & 0.55 & 0.98 \\
CV [\% CP] & 3.21 & 3.44 & 2.56 & 5.95 \\
min [x-fold] & -1.64 & -1.53 & -1.69 & -2.02 \\
max [x-fold] & 1.68 & 1.65 & 1.60 & 2.02 \\
std dev [+/- x-fold] & 1.63 & 1.51 & 1.47 & 1.98 \\
\hline
\end{tabular}

Table 3: Crossing points (CP) data of housekeeping Genes by Best Keeper for $48 \mathrm{hr}$ post transfection.

\begin{tabular}{lcccc}
\hline & Ctbp1 & Gapdh & Prdx1 & Actb \\
\cline { 2 - 5 } $\mathrm{N}$ & 6 & 6 & 6 & 6 \\
\hline geo mean [CP] & 21.00 & 17.87 & 21.71 & 16.85 \\
AR mean [CP] & 21.02 & 17.88 & 21.71 & 16.89 \\
min [CP] & 19.89 & 17.30 & 21.31 & 15.51 \\
max [CP] & 22.14 & 18.42 & 22.14 & 18.22 \\
std dev [+/- CP] & 0.98 & 0.38 & 0.30 & 1.26 \\
CV [\% CP] & 4.65 & 2.13 & 1.37 & 7.47 \\
min [x-fold] & -2.16 & -1.49 & -1.32 & -2.52 \\
max [x-fold] & 2.20 & 1.46 & 1.35 & 2.59 \\
std dev [+/- x-fold] & 1.97 & 1.30 & 1.23 & 2.40 \\
\hline
\end{tabular}


Further data processing involved consideration of $\mathrm{CV}$ and Pearson correlation coefficient $(r)$ of all candidate reference genes. In $4 \mathrm{~T} 1$ cells with $24 \mathrm{hr}$ post-transfection, the Pearson correlation coefficient for all reference genes was quite high which shows a strong resemblance among inter-genes and with BestKeeper index (Table 4). But by taking $\mathrm{CV}$ as criteria for selection of endogenous gene, two genes i.e., Ctbp1 and Actb with the highest variation can be excluded. Of the remaining two genes i.e., Gapdh and Prdx1, minimum variation was shown by $\operatorname{Prd} 1 \times 1$ which suggest the

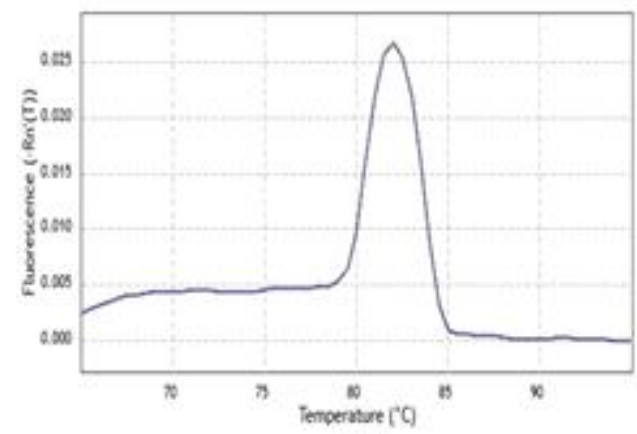

(a)

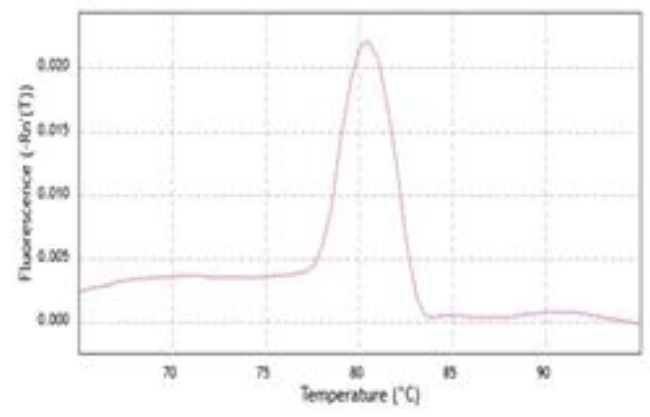

(c) significance of this gene for taking as an endogenous control for qPCR experiments.

In the case of $4 \mathrm{~T} 1$ cells with $48 \mathrm{hr}$ post-transfection, the reference gene having $S D>1$ i.e. Actb was previously eliminated. Further data analysis with Gapdh, Ctbp1 and Prdx 1 was done for their expression stability. Pearson correlation coefficient was high for all these remaining three genes but deviation from the mean (CV) was highest for Ctbp1 which warrants the exclusion of this gene (Table 5). Between Gapdh and Prdx1, the Correlation coefficient was

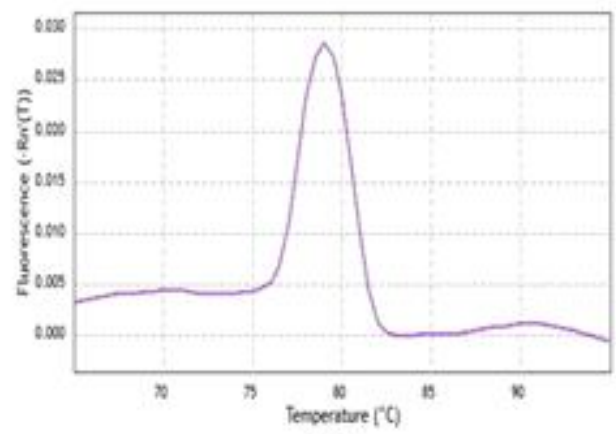

(b)

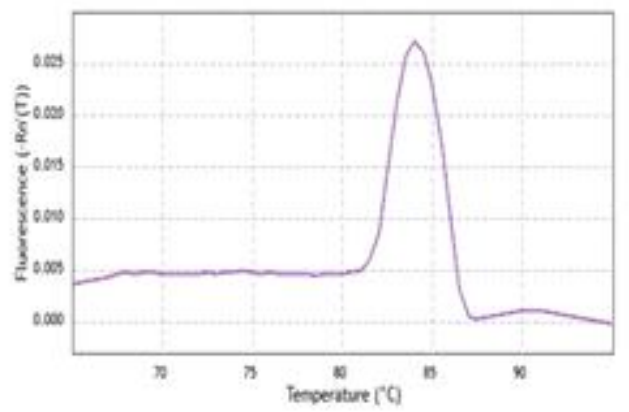

(d)

Fig 2: Single peak observed by each reference gene in Melting Curve Analysis.

(a) Melt Curve of Gapdh.

(b) Melt Curve of Prdx1

(c) Melt Curve of Actb

(d) Melt Curve of Ctbp1
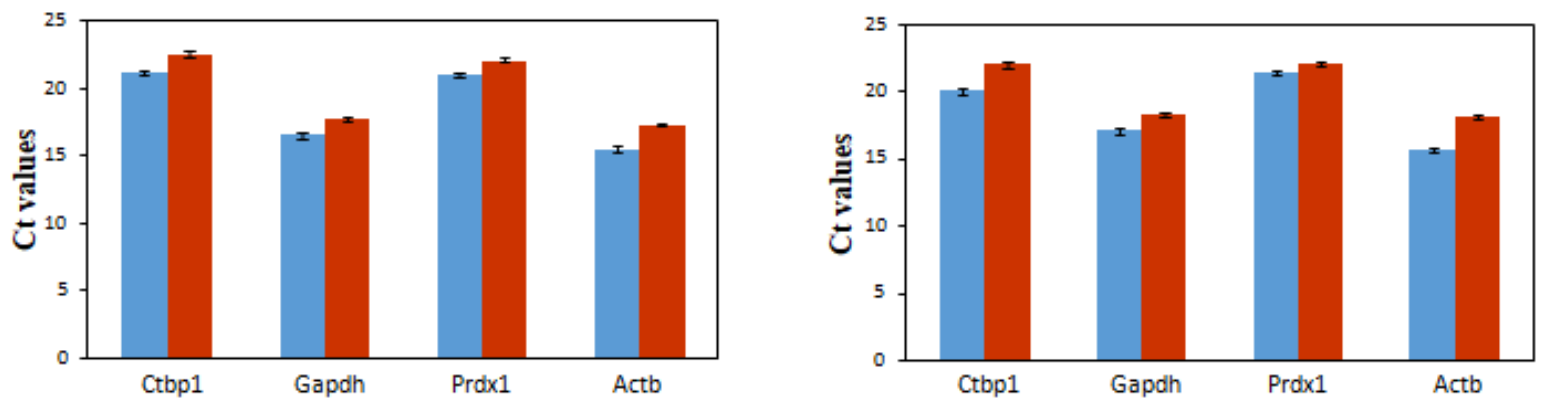

Fig 3: Visualization of reference gene with stable expression in both control (Blue colour) and treatment (Red colour) groups at both 24 and $48 \mathrm{hr}$ post transfection by qPCR.

(a) 24 hour post transfection

(b) 48 hour post transfection 

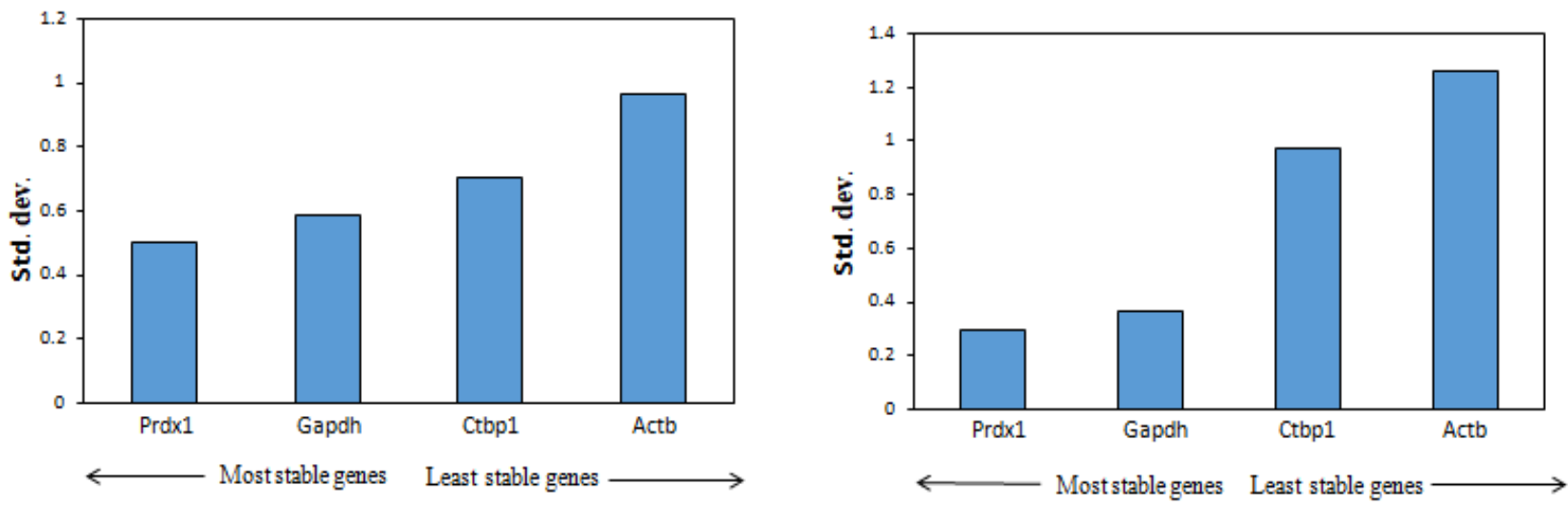

Fig 4: Ranking of most stable to least stable reference genes by BestKeeper.

(a) 24 hour post transfection

(b) 48 hour post transfection

Table 4: Best Keeper correlation analysis ( $24 \mathrm{hr}$ post transfection).

\begin{tabular}{lcccc}
\hline BestKeeper vs. & Ctbp1 & Gapdh & Prdx1 & Actb \\
\hline Coeff. of corr. [r] & 0.997 & 0.994 & 0.978 & 1.000 \\
p-value & 0.001 & 0.001 & 0.001 & 0.001 \\
\hline
\end{tabular}

Table 5: Best Keeper correlation analysis (48hr post transfection).

\begin{tabular}{lllll}
\hline BestKeeper vs. & Ctbp1 & Gapdh & Prdx1 & Actb \\
\hline Coeff. of corr. [r] & 0.999 & 0.965 & 0.974 & 0.998 \\
p-value & 0.001 & 0.002 & 0.001 & 0.001
\end{tabular}

high for both, but Prdx1 due to minimum variation and $p<0.001$ can be considered as a better option among both endogenous genes for qPCR based studies in 4T1 mouse mammary tumor cell line. The ranking of four candidate reference genes on the basis of variation from the most stable to the least stable for both 24 and $48 \mathrm{hr}$ is depicted in Fig 4.

So, in conclusion, three reference genes i.e. Prdx1, Gapdh and Ctbp1 were found to be quite correlated with the BestKeeper index, but by considering all three criteria of selection by BestKeeper algorithm, Prdx 1 showed minimum variation and was found to be ranked at first position by BestKeeper in both time intervals which suggests $\operatorname{Prd} x 1$ to be considered as better internal control gene in 4T1 mouse mammary tumor cell line transfected with CPV2.NS1 for qRT-PCR experiments.

\section{Author contributions}

RA and AKT conceived the idea for the article and prepared the first draft of the manuscript. RA and WAM searched the relevant literature. AT and SS helped in drafting the manuscript. BS and SM contributed by critically revising its contents. All the authors have read and approved the final version of the manuscript and agree to be accountable for its contents.

\section{Funding sources}

This study was supported in part by National Agricultural Innovation Project (Project Code C4/3001) and CAASTACLH, ICAR-IVRI.

\section{Conflict of interest}

The authors declare no conflict of interest.

\section{ACKNOWLEDGEMENT}

We thank Director, IVRI for providing Institute fellowship and contingency for RA (P-2078). We also thank Department of Biotechnology, Government of India (DBT, Gol) for providing fellowship and contingency for WM (DBT Fellow No. DBT/ 2017/IVRI/769).

\section{REFERENCES}

Bao, Z., Huang, Y., Chen, J., Wang, Z., Qian, J., Xu, J., Zhao, Y. (2019). Validation of Reference Genes for Gene Expression Normalization in RAW264.7 Cells under Different Conditions. Biomed Res International. 6131879.

Bhat, A.H., Ganguly, B., Tiwari, A.K., Das, A.K. (2017). Canine Parvovirus NS1 gene and Chicken Anemia VP3 gene induce partial oncolysis of Canine Transmissible Venereal Tumor. Scientific Reports. 7(1): 15419.

Gupta, S.K., Sahoo, A.P., Rosh, N., Gandham, R.K., Saxena, L., Singh, A.K. et al. (2016). Canine parvovirus NS1 induced apoptosis involves mitochondria, accumulation of reactive oxygen species and activation of caspases. Virus Research. 213: 46-61.

Gupta, S.K., Gandham, R.K., Sahoo, A.P., Tiwari, A.K. (2015). Viral genes as oncolytic agents for cancer therapy. Cellular and Molecular Life Science. 72(6): 1073-1094.

Han, L.Q., Yang,G. Y., Zhu, H.S., Wang, Y.Y., Wang, L.F., Guo, Y.J., et al. (2010). Selection and use of reference genes in mouse mammary glands. Genetics and Molecular Research. 9(1): 449-456.

Köhsler, M., Leitsch, D., Müller, N., Walochnik,J. (2020). Validation of reference genes for the normalization of RT-qPCR gene expression in Acanthamoeba spp. Scientific Reports. 10(1): 10362.

Mehta, R., Birerdinc, A., Hossain, N., Afendy,A., Chandhoke, V., Younossi,Z., Baranova, A. (2010). Validation of endogenous reference genes for qRT-PCR analysis of human visceral adipose samples. BMC Mol Biology. 11: 39. 
Pfaffl, M.W., Tichopad, A., Prgomet,C., Neuvians, Y.L. (2004). Determination of stable housekeeping genes, differentially regulated target genes and sample integrity: BestKeeperExcel-based tool using pair-wise correlations. Biotechnology Letters. 26(1): 509-515.

Sahu, A.R., Wani, S.A.,Saxena, S., Rajak, K.K., Chaudhary, D., Sahoo, A.P. et al. (2018). Selection and validation of suitable reference genes for qPCR gene expression analysis in goats and sheep under Peste des petits ruminants virus (PPRV), lineage IV infection. Scientific Reports. 8(1): 15969.

Santra, L., Rajmani, R.S., Kumar, G.V., Saxena, S., Dhara, S.K., Kumar, A. et al. (2014). Non-Structural protein 1 (NS1) gene of Canine Parvovirus-2 regresses chemically induced skin tumors in Wistar rats. Research in Veterinary Science. 97(2): 292-296.

Saxena, L., Kumar, G.R., Saxena, S., Chaturvedi, U., Sahoo, A.P., Singh, L.V. et al. (2013). Apoptosis induced by NS1 gene of Canine Parvovirus-2 is caspase dependent and p53 independent. Virus Research. 173(2): 426-430.
Sleeckx, N., de Rooster, H., Veldhuis Kroeze, E.J., Van Ginneken, C., Van Brantegem., L. (2011). Canine mammary tumours, an overview. Reproduction in Domestic Animal. 46(6): 1112-1131.

Tong, Z., Gao, Z., Wang, F., Zhou, J., Zhang, Z. (2009). Selection of reliable reference genes for gene expression studies in peach using real-time PCR. BMC Mol Biology. 10: 71.

van de Moosdijk, A.A., van Amerongen, R. (2016). Identification of reliable reference genes for qRT-PCR studies of the developing mouse mammary gland. Scientific Reports. 6: 35595.

Xie, F., Xiao, P., Chen, D., Xu, L., Zhang, B. (2012). miRDeepFinder: a miRNA analysis tool for deep sequencing of plant small RNAs. Plant Molecular Biology.

Zaimy, M. A., Saffarzadeh, N., Mohammadi, A., Pourghadamyari,H., Izadi, P., Sarli, A. et al. (2017). New methods in the diagnosis of cancer and gene therapy of cancer based on nanoparticles. Cancer Gene Therapy. 24(6): 233-243. 